\title{
Conformal Degeneracy and Conformal Diffusion of Vesicles
}

\author{
Frank Jülicher, Udo Seifert, and Reinhard Lipowsky \\ Institut für Festkörperforschung, Forschungszentrum Jülich, 52425 Jülich, Germany
}

(Received 19 April 1993)

\begin{abstract}
The shape of vesicles with genus $g=2$, i.e., with two holes or two handles, is studied in the framework of curvature models. These vesicles exhibit a new phase which also persists for higher genus $g>2$. In this phase, the ground state of the vesicle is conformally degenerate even when the volume, the area, and the total mean curvature of the vesicle are kept constant. It is predicted that such vesicles undergo a new type of diffusive motion, termed conformal diffusion, which should be observable in experiments as pronounced shape fluctuations.
\end{abstract}

PACS numbers: $82.70 .-\mathrm{y}, 02.40 .-\mathrm{k}$

One intriguing aspect of lipid vesicles is the need to use concepts of differential geometry in order to explain their behavior as observed under the microscope. It is now generally believed that lipid bilayers, in their liquid state, acquire a shape which minimizes their bending energy and, thus, their curvature energy. In fact, for the case of spherical topology the large variety of observed shapes as well as their transformations can be well understood in terms of a curvature model [1].

Recently, vesicles of toroidal topology have been found experimentally [2] which had been previously predicted in theoretical work [3-6]. The new feature of toroidal shapes not present for shapes of spherical topology is the degeneracy of the shape of minimal curvature energy with respect to conformal transformations of the threedimensional embedding space $[4,7]$. However, since this degeneracy is broken by the presence of physical constraints such as the fixed area to volume ratio of vesicles, it has no observable consequences for toroidal vesicles and the ground state of these vesicles is unique in any realistic model [6].

The purpose of this paper is to show that for genus-2 vesicles, i.e., for vesicles with two holes or two handles, qualitatively new behavior emerges. For these vesicles, as well as for all higher genus vesicles, the ground state is degenerate with respect to conformal transformations for a certain range of shapes, even if one takes into account the constraints on the area to volume ratio and the additional constraint arising from the fact that the membrane is a bilayer. Thus we predict that conformal modes become visible in the microscope as pronounced shape fluctuations for vesicles with genus $g \geq 2$. Moreover, we determine the phase diagram for genus- 2 vesicles using a combination of direct numerical minimization for nonaxisymmetric shapes, conformal transformations, and symmetry considerations.

The shape fluctuations which correspond to conformal modes are quite different from shape fluctuations of vesicles of spherical topology (and genus-1 vesicles) where bending modes with finite energy (i.e., nonzero restoring force) are thermally excited. In contrast, the conformal modes lead to a diffusion process in shape space along a path of conformally degenerate shapes. In analogy with translational and rotational diffusion, this process will be called conformal diffusion; an example is shown in Fig. 1.

To derive these results, we first recall the Willmore problem [8]: Determine the surface which minimizes the curvature energy

$$
G \equiv \frac{\kappa}{2} \oint(2 H)^{2} d A,
$$

where $H$ is the mean (extrinsic) curvature and $\kappa$ is the bending rigidity. For genus-0, the solution is known to be the sphere with $G=G_{0} \equiv 8 \pi \kappa$, while for genus-1 the solution is conjectured to be the Clifford torus (and its conformal transformations) with $G=G_{1} \equiv(\pi / 2) 8 \pi \kappa$ [8]. For genus-2, there is a mathematical conjecture by Kusner [9] that the Willmore problem is solved by the Lawson surface $L$ shown in Fig. $2[10,11]$. By numerically minimizing a discretized version of the energy $G$, Hsu, Kusner, and Sullivan found the Lawson surface $L$ to be a minimum of $G$ with $G=G_{2} \simeq 1.742 \times 8 \pi \kappa[12]$. To obtain a numerical representation of a genus- 2 Willmore surface, we also developed an algorithm for minimizing the bending energy $G$ for a triangulated surface [13]. We find the Lawson surface $L$ as shown in Fig. 2 with $G_{2} \simeq(1.75 \pm 0.01) \times 8 \pi \kappa$, as a minimum of our algorithm, in full agreement with the results of Hsu, Kusner,

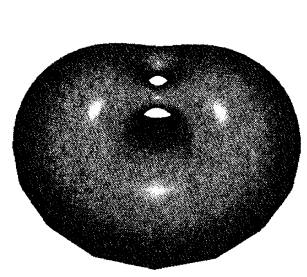

(a)

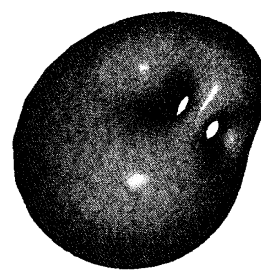

(b)

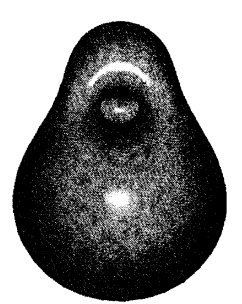

(c)
FIG. 1. Three different shapes which all minimize the curvature energy $G$ for fixed reduced volume $v \simeq 0.78$ and $m \simeq 1.027$. These shapes represent an example for a conformal mode. The shapes (a) and (c) are the two special shapes along this conformal mode which both possess one symmetry plane, while shape (b) has no symmetry plane. 


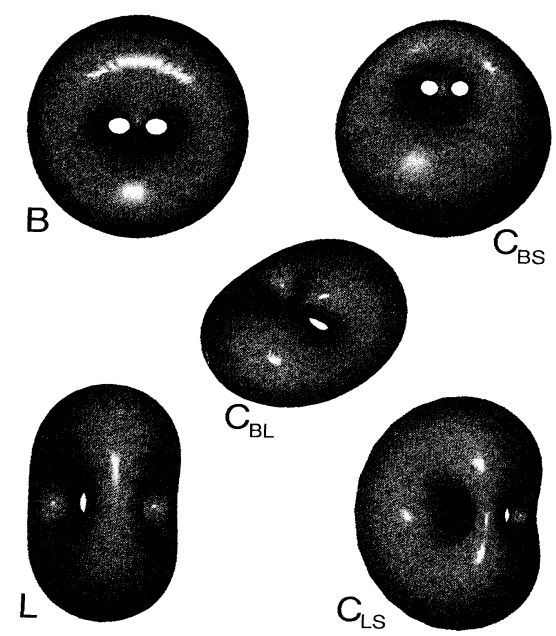

FIG. 2. Willmore surfaces with energy $G=G_{2} \simeq 1.75$ $\times 8 \pi \kappa$. These shapes correspond to points at the boundary of the region $W$ : The $\mathcal{D}_{3 h}$ symmetric Lawson surface $L$; the $\mathcal{D}_{2 h}$ symmetric button surface $B$; and examples of $\mathcal{C}_{2 v}$ symmetric shapes along the lines $C_{B S}, C_{B L}$, and $C_{L S}$, respectively; compare Fig. 3.

and Sullivan. This surface has a threefold symmetry axis and an additional mirror symmetry plane and, thus, $\mathcal{D}_{3 h}$ symmetry in the Schönflies nomenclature, which we will use further on.

We now use the property that the bending energy $G$ of any shape is invariant under special conformal transformations (SCT's) of the three-dimensional embedding space $[7,8]$ to generate a continuous space $\mathcal{W}$ of Willmore surfaces with the same energy $G=G_{2}$, but with symmetry properties which are different from those of the Lawson surface. A SCT, which can be parametrized by a vector $\mathbf{a}=\left(a_{x}, a_{y}, a_{z}\right)$, transforms any point $\mathbf{R}$ of the surface to $\mathbf{R}^{\prime}$ with $\mathbf{R}^{\prime}=\left(\mathbf{R} / R^{2}+\mathbf{a}\right) /\left(\mathbf{R} / R^{2}+\mathbf{a}\right)^{2}$. Applying SCT's to the Lawson surface $L$, the three-dimensional space $\mathcal{W}$ of Willmore surfaces which can be parametrized by the vector $\mathbf{a}$ is found. Consequently, the ground state for the energy $G$ is threefold degenerate for genus-2 [14].

For real vesicles, both the total area $A \equiv 4 \pi R_{0}^{2}$, as well as the enclosed volume $V$, are fixed quantities. Moreover, the vesicle membrane is a bilayer which leads to a further constraint on the area difference of the two monolayers. These constraints imply that both the reduced volume $v \equiv V /\left[4 \pi(A / 4 \pi)^{3 / 2} / 3\right]$ and the reduced mean curvature $m \equiv \oint d A H /(A / 4 \pi)^{1 / 2}$ are constant (at constant temperature). Thus, the physical state of the genus- 2 vesicle corresponds to the shape of minimal curvature energy $G$ for given $v$ and $m$.

A part of this phase diagram is obtained by projecting the three-dimensional space $\mathcal{W}$ onto the $(v, m)$ plane. This leads to a region $W$ in the phase diagram where the ground state is indeed given by the Willmore surfaces. Since the space $\mathcal{W}$ is three-dimensional while the $(v, m)$ plane is two dimensional, the ground state within the region $W$ is one dimensional degenerate. A quantitative expression for the conformal mode which corresponds to this degeneracy can be obtained by studying SCT's with small $|\mathbf{a}|<<1$. A SCT acting on a surface with initial values $v=v_{1}$ and $m=m_{1}$ generates a new shape with $v=v_{1}(\mathbf{a})$ and $m=m_{1}(\mathbf{a})$ given by $v_{1}(\mathbf{a})=v_{1}[1+$ $\left.A_{\alpha}^{(v)} a_{\alpha}+O\left(\mathbf{a}^{2}\right)\right]$ and $m_{1}(\mathbf{a})=m_{1}\left[1+A_{\alpha}^{(m)} a_{\alpha}+O\left(\mathbf{a}^{2}\right)\right]$. The vector coefficients $\mathbf{A}^{(v)}$ and $\mathbf{A}^{(m)}$ can be expressed as $\mathbf{A}^{(v)} \equiv 6\left(\mathbf{R}^{A}-\mathbf{R}^{V}\right)$ and $\mathbf{A}^{(m)} \equiv 2\left(\mathbf{R}^{A}-\mathbf{R}^{M}\right)$ by the center of area $\mathbf{R}^{A} \equiv \oint d A \mathbf{R} / A$, the center of volume $\mathbf{R}^{V} \equiv \int d V \mathbf{R} / V$, and the center of mean curvature $\mathbf{R}^{M} \equiv \oint d A H \mathbf{R} / \oint d A H$ [15]. Thus, the conformal mode which conserves both $v$ and $m$ can be identified as the SCT with a obeying the differential equation

$$
d \mathbf{a} / d s=\mathbf{A}^{(v)} \times \mathbf{A}^{(m)},
$$

where $s$ parametrizes the path in the space $\mathcal{W}$. In general, this conformal mode a $(s)$ which solves (2) destroys all symmetry planes of the shapes in the region $W$. However, along the path $\mathbf{a}(s)$ which is a closed loop within $\mathcal{W}$, there are two different shapes which have one symmetry plane left. The shapes shown in Fig. 1 represent the conformal mode with $(v, m)=(0.78,1.027)$ and have been obtained by numerical integration of (2).

The boundary of the region $W$ in the phase diagram is most easily determined numerically by first introducing the button surface $B$ shown in Fig. 2. This is another highly symmetric Willmore surface which is conformally equivalent to the Lawson surface $L$ and which was already found by $\mathrm{Hsu}$, Kusner, and Sullivan [12]. The surface $B$ with $(v, m) \simeq(0.66,1.084 \times 4 \pi)$ has three orthogonal mirror symmetry planes, i.e., symmetry $\mathcal{D}_{2 h}$. We now apply SCT's to the button surface. If the $x-y$ plane is chosen to be the midplane of the disk and the centers of the two holes lie on the $x$ axis, a SCT with $\mathbf{a}=\left(0,0, a_{z}\right)$ breaks the symmetry with respect to the $x-y$ plane and generates the line $C_{B L}$ with shapes of $\mathcal{C}_{2 v}$ symmetry; see Fig. 2. For $a_{z} \simeq 3.4 / R_{0}$, the $\mathcal{D}_{3 h}$-symmetric Lawson surface $L$ with $(v, m) \simeq(0.67,1.037 \times 4 \pi)$ is reached. A further increase in $a_{z}$ breaks the threefold symmetry, generating the line $C_{L S}$ with $\mathcal{C}_{2 v}$-symmetric shapes; see Fig. 2. For $a_{z} \simeq 15.5 / R_{0}$, the shape along $C_{L S}$ approaches a sphere $S$ with two infinitesimal handles at $(v, m)=(1,4 \pi)$. The line $C_{B S}$ can be generated from the button surface by breaking the $x-z$ symmetry plane with $\mathbf{a}=\left(0, a_{y}, 0\right)$. A typical shape along this line is also shown in Fig. 2. These shapes again approach a sphere $S$ with two infinitesimal handles for $a_{y} \simeq 0.8 / R_{0}$ at $(v, m)=(1,4 \pi)$. The shapes along $C_{B S}, C_{B L}$, and $C_{L S}$ constitute the boundary of the region $W$ since these shapes have at least two mirror planes, i.e., $\mathcal{C}_{2 v}$ symmetry, which implies that $\mathbf{A}^{(v)}$ and $\mathbf{A}^{(m)}$ are parallel and there is no conformal mode as follows from (2) [16].

Outside of $W$, the shapes of minimal energy are no longer Willmore surfaces with constant energy $G=G_{2}$, 


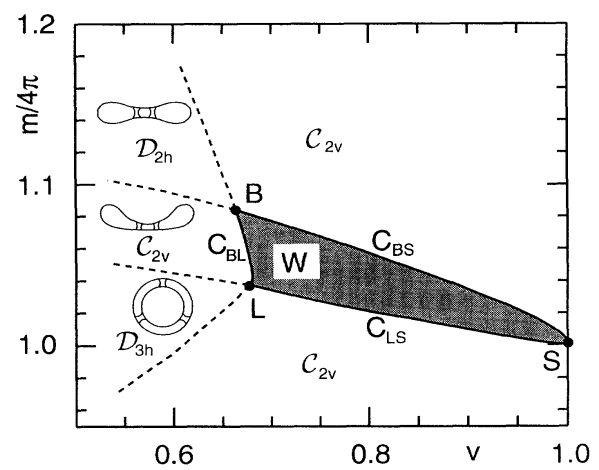

FIG. 3. Phase diagram for genus- 2 vesicles. Within the phase $W$, the ground state is conformally degenerate. This region is bounded by the lines $C_{B S}, C_{B L}$, and $C_{L S}$. The Lawson surface $L$ and the button surface $B$ are special points at the boundary of $W$. Adjacent to $W$, five different regions exist. The symmetries of the shapes within these regions are indicated. Cartoons characterize the shapes within three regions.

but rather shapes with a larger energy $G=G(v, m)$, which can also be classified by their symmetry properties. Any shape on a sheet of stationary shapes where generically $\partial G / \partial v \neq 0$ and $\partial G / \partial m \neq 0$ must fulfill the condition that $\mathbf{A}^{(v)}$ is parallel to $\mathbf{A}^{(m)}$. Otherwise one could find a shape nearby using conformal transformations which has the same $v$ and $m$ but lower energy [15]. By symmetry, $\mathbf{A}^{(v)}$ is parallel to $\mathbf{A}^{(m)}$ for any shape with at least two symmetry planes. Thus, the ground state outside of $W$ should have at least $\mathcal{C}_{2 v}$ symmetry. The topology of the phase diagram of genus- 2 vesicles as shown in Fig. 3 then follows from the symmetry properties of the Willmore shapes.

It is instructive to compare the genus-2 phase diagram with the phase diagram of toroidal (or genus-1) shapes shown in Fig. 4 [6]. Apart from a large region of nonaxisymmetric shapes this phase diagram contains three regions of axisymmetric shapes: (i) a region of discoid tori, (ii) a region of sickle-shaped tori, and (iii) a region of toroidal stomatocytes. All regions are separated by continuous phase boundaries. The genus-1 Willmore surfaces form a one-dimensional space and exist along a line $W$, which starts with the Clifford torus $C L$ and ends up at $(v, m)=(1,4 \pi)$ with a sphere $S$ with an infinitesimal handle.

Comparison of Figs. 4 and 3 shows that the two phase diagrams for genus- 1 and genus- 2 are quite similar. The three axisymmetric families of genus-1 shapes, the discoid tori, toroidal stomatocytes, and the sickle-shaped tori, correspond to the genus- 2 shapes with $\mathcal{D}_{2 h}, \mathcal{C}_{2 v}$, and $\mathcal{D}_{3 h}$ symmetry, respectively. The corresponding shapes indeed look very similar even though the additional hole of the genus- 2 shapes necessarily breaks the axisymmetry. Likewise, the line $W$ of genus-1 Willmore tori in

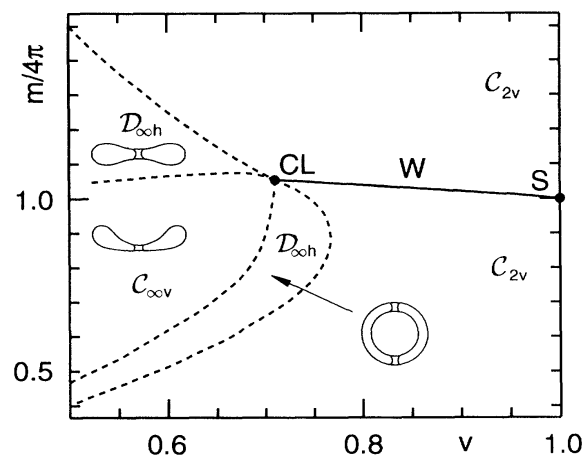

FIG. 4. Phase diagram for genus-1 vesicles. Three regions of axisymmetric shapes exist: (i) $\mathcal{D}_{\infty h}$ symmetric discoid tori, (ii) $\mathcal{D}_{\infty h}$ symmetric sickle-shaped tori, and (iii) $\mathcal{C}_{\infty v}$ symmetric toroidal stomatocytes. The large region of nonaxisymmetric shapes has $\mathcal{C}_{2 v}$ symmetry. The line $W$ denotes the Willmore surfaces starting at the Clifford torus $C L$.

Fig. 4 corresponds to the region $W$ for genus- 2 vesicles in Fig. 3.

The present reasoning can be extended to vesicles with higher genus. Regions with conformally degenerate ground state exist for any genus $g \geq 2$. For $g>2$, the functional (1) possesses even more than one stationary point [17]. Consequently, there will be several regions of one-dimensional degenerate ground states for higher genus vesicles. Discontinuous transitions together with regions of metastability are then likely to occur.

Similar results are found if one relaxes the hard constraint on $m$ and considers the area difference elasticity model (ADE model) with energy $W \equiv G+\kappa \alpha(m-$ $\left.m_{0}\right)^{2} / 2$. Here, $m_{0}$ is the equilibrium value of the (scaled) area difference and $\alpha$ is the ratio of two elastic moduli with $\alpha \simeq 1$ [18]. In the $\left(v, m_{0}\right)$ plane of this model, the region $W$ with conformally degenerate ground state has the same shape as in the $(v, m)$ plane shown in Fig. 3. Outside of $W$, the location of the phase boundaries depends on $\alpha$. For small enough $\alpha$, we expect first-order transitions to occur.

Genus-2 vesicles have recently been observed experimentally [2]. The observed genus-2 discocyte with $\mathcal{D}_{2 h}$ symmetry can be directly located in the phase diagram for genus-2 shapes shown in Fig. 3. According to our theory, a ground state with three symmetry planes cannot be degenerate. Consequently, this shape does not belong to the degenerate phase $W$ and should not exhibit conformal diffusion in agreement with the experimental observations.

For a crucial test of the results presented here, one control parameter, such as the temperature, should be varied systematically. Such a temperature change corresponds to a path in the phase diagram. Starting from a genus-2 discocyte as observed in Ref. [2], a decrease in temperature will increase $v$ which should eventually lead 
into the phase $W[19]$. The onset of conformal diffusion should then be observed as soon as the temperature trajectory crosses the boundary to the region $W$. Within $W$, the typical diffusion time in the shape space should be of the order of $t_{c f}=\eta R_{0}^{3} / T$ for a solvent of viscosity $\eta$ at temperature $T$, as follows from dimensional analysis. Note that this time scale is significantly longer than the time scale $t_{b}=\eta R_{0}^{3} / \kappa$ for long-wavelength bending fluctuations since $\kappa / T \simeq 25$ for phospholipid membranes.

The existence of the phase $W$ is strongly dependent on the specific form of the local curvature energy as given by (1). The observation of conformal diffusion would therefore directly confirm this form. Even though one expects higher order corrections to this energy, the amplitude of these terms should scale like $\sim d / R_{0} \simeq 10^{-3}$, where $d$ is the thickness of the bilayer and $R_{0}$ the size of the vesicle. Thus, these terms are not expected to suppress the conformal diffusion process proposed here.

In summary, we have shown that genus- 2 vesicles exhibit a new phase characterized by conformal diffusion which makes conformal transformations visible in the microscope.

We thank D. Bensimon and X. Michalet for stimulating interactions and W. Schnitzler for his help in developing the algorithm used in this paper.

[1] For a review, see R. Lipowsky, Nature (London) 349, 475 (1991).

[2] B. Fourcade, M. Mutz, and D. Bensimon, Phys. Rev. Lett. 68, 2251 (1992).

[3] Ou-Yang Zhong-can, Phys. Rev. A 41, 4517 (1990).

[4] U. Seifert, Phys. Rev. Lett. 66, 2404 (1991).

[5] B. Fourcade, J. Phys. II (France) 2, 1705 (1992).
[6] F. Jülicher, U. Seifert, and R. Lipowsky (to be published).

[7] B. Duplantier, Physica (Amsterdam) 168A, 179 (1990); B. Duplantier, R.E. Goldstein, V. Romero-Rochin, and A.I. Pesci, Phys. Rev. Lett. 65, 508 (1990).

[8] T.J. Willmore, Total Curvature in Riemannian Geometry (Ellis Horwood, Chichester, 1982).

[9] R. Kusner, Pacific J. Math. 138, 317 (1989).

[10] H.B. Lawson, Ann. Math. 92, 335 (1970).

[11] H. Karcher, U. Pinkall, and I. Sterling, J. Diff. Geom. 28, 169 (1988).

[12] L. Hsu, R. Kusner, and J. Sullivan, J. Exp. Math. 1, 191 (1992).

[13] F. Jülicher and W. Schnitzler (unpublished).

[14] The corresponding space $\mathcal{W}$ of genus- 1 surfaces is only one dimensional. The reduction of dimensionality is a consequence of the high degree of symmetry of the Clifford torus. This fact was overlooked in Ref. [4], where a degeneracy of the ground state of genus-1 vesicles was claimed erroneously even in the presence of constraints.

[15] U. Seifert, J. Phys. A 24, L573 (1991).

[16] Strictly speaking, there are also lines within $W$, where shapes with $\mathcal{C}_{2 v}$ and $\mathcal{C}_{3 v}$ symmetry exist. The boundary of $W$ is defined by all those lines which bound the largest area in the $(v, m)$ plane.

[17] This follows from the fact that there are several minimal surfaces without self-intersections in $S^{3}$ which projected onto $R^{3}$ make the curvature energy (1) stationary; see [10-12].

[18] U. Seifert, L. Miao, H.-G. Döbereiner, and M. Wortis, in The Structure and Conformation of Amphiphilic Membranes, edited by R. Lipowsky, D. Richter, and K. Kremer (Springer, Berlin, 1992), p. 93; B. Božič, S. Svetina, B. Žekš, and R.E. Waugh, Biophys. J. 61, 963 (1992); W. Wiese, W. Harbich, and W. Helfrich, J. Phys. Condens. Matter 4, 1647 (1992).

[19] For symmetric bilayers, temperature trajectories lie on hyperbolas $m=$ const $/ v$; see U. Seifert, K. Berndl, and R. Lipowsky, Phys. Rev. A 44, 1182 (1991). 


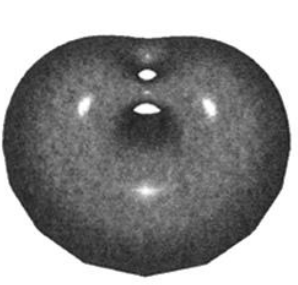

(a)

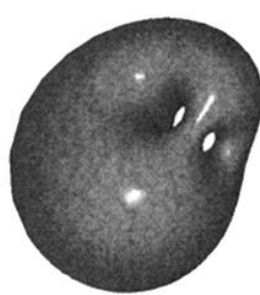

(b)

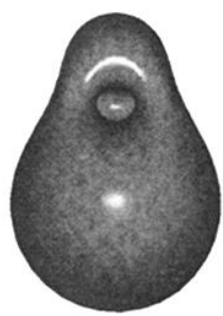

(c)

FIG. 1. Three different shapes which all minimize the curvature energy $G$ for fixed reduced volume $v \simeq 0.78$ and $m \simeq 1.027$. These shapes represent an example for a conformal mode. The shapes (a) and (c) are the two special shapes along this conformal mode which both possess one symmetry plane, while shape (b) has no symmetry plane. 


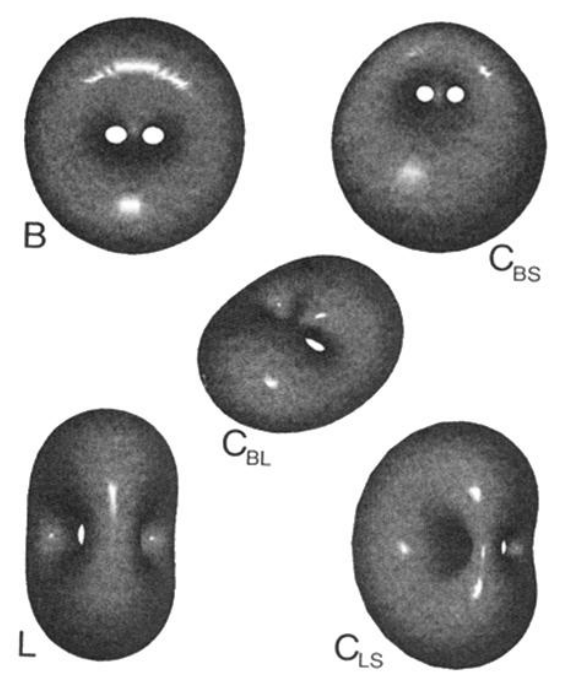

FIG. 2. Willmore surfaces with energy $G=G_{2} \simeq 1.75$ $\times 8 \pi \kappa$. These shapes correspond to points at the boundary of the region $W$ : The $\mathcal{D}_{3 h}$ symmetric Lawson surface $L$; the $\mathcal{D}_{2 h}$ symmetric button surface $B$; and examples of $\mathcal{C}_{2 v}$ symmetric shapes along the lines $C_{B S}, C_{B L}$, and $C_{L S}$, respectively; compare Fig. 3. 